\title{
Direct hyperpolarization of micro- and nanodiamonds for bioimaging applications - Considerations on particle size, functionalization and polarization loss
}

\section{Journal Article}

Author(s):

Kwiatkowski, Grzegorz; Jähnig, Fabian; Steinhauser, Jonas; Wespi, Patrick; Ernst, Matthias (D); Kozerke, Sebastian

Publication date:

2018-01

Permanent link:

https://doi.org/10.3929/ethz-b-000217958

Rights / license:

Creative Commons Attribution-NonCommercial-NoDerivatives 4.0 International

Originally published in:

Journal of Magnetic Resonance 286, https://doi.org/10.1016/j.jmr.2017.11.007

\section{Funding acknowledgement:}

170729 - Integrated system for in operando characterization and development of portable breath analyzers (SNF) 149707 - Method Development for Dissolution Dynamic Nuclear Polarization (SNF)

169879 - Method Development in Solid-State NMR and Dissolution DNP (SNF)

163243 - Multifunctional nanoparticles for targeted theranostics (SNF) 


\section{Exploiting endogenous surface defects for dynamic nuclear polarization of}

\section{silicon micro- and nanoparticles}

Grzegorz Kwiatkowski ${ }^{1}$, Yevhen Polyhach ${ }^{2}$, Fabian Jähnig ${ }^{2}$, Toni Shiroka ${ }^{3,4}$, Fabian H.L. Starsich ${ }^{5}$,

Matthias Ernst ${ }^{2}$, Sebastian Kozerke ${ }^{1}$

${ }^{1}$ Institute for Biomedical Engineering, University and ETH Zurich, Switzerland;

${ }^{2}$ Laboratory of Physical Chemistry, ETH Zurich, Switzerland

${ }^{3}$ Laboratory for Solid State Physics, ETH Zurich, Switzerland

${ }^{4}$ Paul Scherrer Institut, Villigen PSI, Switzerland

${ }^{5}$ Particle Technology Laboratory, Institute of Process Engineering, ETH Zurich, Switzerland

Submission: Journal of Physical Chemistry C

Mailing Address: $\quad$ Sebastian Kozerke, PhD

Institute for Biomedical Engineering

University and ETH Zurich

Gloriastrasse 35

8092 Zurich

Tel.: + + 41446323549

FAX: $\quad+41446321193$

Email: kozerke@biomed.ee.ethz.ch 


\begin{abstract}
Micro- and nanoparticles of elemental, crystalline silicon represent an attractive target for a wide range of applications spanning from quantum computing to contrast agents for biomedical imaging applications. To overcome the low sensitivity of the ${ }^{29} \mathrm{Si}$ nuclei in magnetic resonance, dynamic nuclear polarization (DNP), which exploits the endogenous surface defects as a source of polarization, can be used to temporarily boost nuclear polarization of the ${ }^{29} \mathrm{Si}$ spin bath. In the present work we have assessed a number of commercially available silicon micro- and nanoparticles concerning properties and characteristics under DNP conditions. It has been found that optimal physical and chemical conditions, including surfacedefect concentration adjusted to the particle size, are necessary to achieve a high level of polarization enhancement.
\end{abstract}




\section{Introduction}

Nuclear Magnetic Resonance (NMR) has been used for decades ${ }^{1}$ to study the structure and dynamics of silicon-based materials. The interest in silicon NMR has recently been renewed due to possible applications of crystalline silicon in quantum computing, both as a $\operatorname{target}^{2}$ or as a host ${ }^{3-5}$ for other nuclei. Naturally low-abundant ${ }^{29} \mathrm{Si}$ (natural abundance of $4.7 \%$, spin$1 / 2$ ) embedded in a crystalline core of spin-0 particles (silicon-28 and silicon-30) constitutes a well-defined spin-system, characterized by long spin-lattice relaxation times, which in some cases can be up to a few hours ${ }^{6,7}$. In addition, the weak nuclear dipole-dipole coupling between the dilute spins results in exceptionally long decoherence times, up to a few seconds $s^{4,8,9}$. Nonetheless, the application of ${ }^{29} \mathrm{Si}$ NMR to studying silicon-based materials suffers from its intrisically low detection sensitivity, a common problem for low-gamma nuclei. Recent advances in NMR hyperpolarization have demonstrated the possibility to enhance the ${ }^{29} \mathrm{Si}$ signal by exploiting surface defects for direct dynamic nuclear polarization ${ }^{10}$ (DNP). Such an approach results in a boost of the available polarization by up to a few orders of magnitude. The time span during which the enhanced magnetization can be stored is intrinsically given by the nuclear $T_{1}$ relaxation time which, in the case of crystalline silicon, can be up to a few hours. Combining the long nuclear relaxation times of silicon with hyperpolarization for enhanced polarization and subsequent detection, is an attractive feature with a potentially wide range of applications. In particular, crystalline silicon in the form of micro- and nanoparticles can be used as a background-free MRI contrast agent. ${ }^{11-15}$ As such, direct MR detection of hyperpolarized micro- and nanoparticles based on crystalline silicon has shown promising results, with the possibility of signal detection beyond one hour after administration of the particles, which is much longer than any times reported for other hyperpolarized biomolecular probes ${ }^{16}$. 
Nanoscale silicon can be obtained using numerous techniques, including solution synthesis ${ }^{17}$, spark discharge ${ }^{18}$, laser-stimulated etching ${ }^{19,20}$, plasma synthesis ${ }^{21,22}$ or ball milling ${ }^{23}$. The application of different production approaches results in different physical and chemical properties of the final materials ${ }^{24}$, in particular, crystallinity, contamination with metal ions, surface oxidation and particle-size distribution. As such, these properties are likely to affect the ability of the silicon particles to be dynamically polarized and hence their usability for NMR/MRI applications.

The aim of the present work was to systematically examine the properties of a range of commercially available samples of silicon particles with respect to their performance for direct DNP application and to discuss the possible optimization routes to maximize the enhancement of ${ }^{29}$ Si polarization.

\section{Hyperpolarization of ${ }^{29}$ Si nuclei}

Hyperpolarization is a general term characterizing several different physical phenomena that allow a temporal enhancement of nuclear spin order. As a result, the spin polarization of the nuclear spins is increased beyond its thermal equilibrium value. The absolute enhancement, defined as the ratio between the enhanced polarization and its value at thermal equilibrium, varies between a few to a few tens of thousands depending on the particular scheme of hyperpolarization and on the system used. Several means of hyperpolarization have been developed $^{25}$, which exploit different properties of the spin system (phase of the substrate, presence of a structural defect or host molecules). Among different means to polarize nuclear spins, optical pumping, chemical reaction or direct transfer of angular momentum from electron- to nuclear spins are the most common. The latter benefit from a large difference in thermal polarization between nuclear spins (typically less than $1 \%$ at cryogenic temperatures and static magnetic fields of around $10 \mathrm{~T}$ ) and electron spins (typically larger than $90 \%$ at 
cryogenic temperatures) which is referred to as $\mathrm{DNP}^{26}$. The transfer of the angular momentum is facilitated by continuous irradiation of the electron-spin system with a microwave frequency close to its Larmor frequency, resulting in partial saturation of the electron-spin bath. The transitory non-Boltzmann distribution of the electron-spin system is then partially transferred to the nuclear-spin system through the hyperfine interaction (magnetic interaction between an electron and a nuclear spins) ${ }^{27}$. In most cases, the source of the unpaired electron comes from doping the sample with exogenous radicals. This means that the sample to be polarized has to be mixed with a glassing agent that carries the dispersed radical. Crystalline silicon offers a possibility to exploit the endogenous lattice defects for DNP, thus alleviating the need to dope the sample with additional radicals and/or glassing agents. The paramagnetic centres in crystalline silicon can be created in several ways $^{28,29}$, including surface oxidation, applying mechanical stress, electrical discharge, exposure to ionizing radiation or doping with metal ions. In particular, the surface defects ${ }^{30}$, which arises due to crystalline lattice mismatch between the elemental silicon core and its oxidized $\left(\mathrm{SiO}_{2}\right)$ top layer have been used for DNP since the first reports of hyperpolarization of silicon ${ }^{31}$. Surface paramagnetic centres can be used to polarize nuclei located at the surface $^{32}$ or within the dispersing solvent ${ }^{33,34}$. The surface defects are enclosed at some depth below the material's surface, hence being protected from the external environment ${ }^{35,36}$. As such, the surface defects are exceptionally stable over a long period of time, resulting in a sample that can be repolarized many times without any changes in its properties. However, as the oxidation of the surface is difficult to control, doping of the silicon with phosphorus offers an alternative route for direct $\mathrm{DNP}^{37,38}$. In such a case, photoexcitation of electrons to the conduction band by band-gap laser illumination can be used to modulate electronic $T_{1}$ relaxation and hence affect the efficiency of the polarization transfer ${ }^{39,40}$. Lastly, by dispersing 
the sample in a glassing agent, the fast polarization of matrix protons can be used to polarize ${ }^{29} \mathrm{Si}$ nuclei indirectly through so-called cross polarization ${ }^{41}$. Such an approach is particularly attractive to study surface phenomena, but fails to provide significant enhancement for bulk

${ }^{29} \mathrm{Si}$ nuclei due to weak dipole-dipole ${ }^{1} \mathrm{H}-{ }^{29} \mathrm{Si}$ coupling with the surface protons. Similarly, doping the sample with an exogenous radical was used to directly enhance the ${ }^{29} \mathrm{Si}$ polarization in a suspension of small, nanometre-size silicon particles, which exhibited an insufficient endogenous surface defect concentration ${ }^{42,43}$.

\section{Experimental section}

\section{Materials}

In total, seven samples of silicon powder were included in this study (Table 1). All samples were obtained commercially with some processing being done in house. Samples AA 1-20, AA 1-5, AA 100 and AA 50 were purchased from Alfa Aesar (Karlsruhe, Germany), samples US 13 and US 20-30 were purchased from US Nano Research (Houston, TX, USA) and sample SiP was obtained from Sigma-Aldrich (product no 647799, Buchs, Switzerland). Sample SiP was obtained in the form of a wafer and subsequently milled into a powder. The whole wafer was first crushed in a mortar and further milled in an ethanol solution for $4 \mathrm{~h}$ using a planetary ball mill. A successive X-ray fluorescence measurement confirmed the absence of contamination from the zirconia balls. For all other samples, the silicon powder was used as obtained from the manufacturer and stored in an air-tight container. No special precautions were taken to prevent air exposure. At the same time, no degradation or change in any of the material properties was observed over a period of 10 months. The production scheme (Table 1) for each sample is described based on the information provided by the manufacturer. Size separation of sample AA 1-20 was obtained by repeated centrifugation and redispersion ${ }^{44}$ in ethanol. The size distribution was confirmed with dynamic light scattering (Zetasizer Nano 
$Z$, Malvern), resulting in samples with the following size distributions: $10 \pm 1,1.5 \pm 0.4,1.1 \pm$ $0.3,0.71 \pm 0.21,0.53 \pm 0.19,0.41 \pm 0.13$ all in $\mu \mathrm{m}$. For comparison between different samples, the sample AA 1-20 without size separation was used.

\section{Hyperpolarization}

A home-built polarizer operating at $B_{0}=3.4 \mathrm{~T}$ and at temperature of about $3.5 \mathrm{~K}$ was used ${ }^{45}$. Depending on the size of the particles, between 60 and $100 \mathrm{mg}$ of sample powder was tightly packed and enclosed in a polytetrafluoroethylene (PTFE) cup. A home-built probe with a solenoid coil wound around the sample cup was used. The microwave field (ELVA-1 VCOM10/94/200-DD, max power $200 \mathrm{~mW}$ ) was delivered by direct irradiation from the waveguide elbow $^{45}$. The sample was inserted into a warm cryostat and cooled gradually over ca. 45 min. During the irradiation period, the samples were continuously immersed in liquid helium to avoid spurious effects of the temperature fluctuation. Microwave irradiation for polarization build up was started earliest $30 \mathrm{~min}$ after cryostat cooling to allow for temperature stabilization. The absolute value of polarization in the solid state was obtained by comparing the integrated signal intensity of the hyperpolarized sample with the thermal-equilibrium signal of $100 \mathrm{mg}$ fully ${ }^{29} \mathrm{Si}$ labeled silicon at $T=295 \mathrm{~K}$ (99\% of ${ }^{29} \mathrm{Si}$, Isofelx, Moscow, Russia). Thermal polarization was measured using the same set-up as for DNP experiments. In the latter case, because of the long relaxation time at low temperature, the thermal reference signal was acquired at room temperature. The correction for the change in signal intensity due to the temperature difference (Boltzmann distribution) and the isotope composition were accounted for. Nonetheless, a relative error of approximately $30 \%$ is expected in the absolute polarization calculation, due to the uncertainty in the excitation pulse flip angle at $3.4 \mathrm{~K}$ and room temperature. For experiments with microwave field modulation, a symmetric 
ramp function with a modulation frequency up to $10 \mathrm{kHz}$ and a modulation depth up to $300 \mathrm{MHz}$ was used.

\section{Room-temperature nuclear relaxation of silicon}

Due to proximity of the MR imaging system to the polarizing set-up, room-temperature ${ }^{29} \mathrm{Si}$ nuclear relaxation has been recorded at 9.4T. The relaxation times at room temperature were measured using an upgraded version of the polarizer with a probe with a custom-design, quasi-cavity structure ${ }^{45}$. After $24 \mathrm{~h}$ of continuous polarization at a temperature of $T=3.4 \mathrm{~K}$, the samples were taken out of the polarizer and immediately transferred to the front of a horizontal 9.4 T imaging system (Bruker BioSpin, Ettlingen, Germany). The relaxation data were recorded with a home-built transmit-receive, 3-loop solenoid coil (10 $\mathrm{mm}$ diameter).

\section{Electron paramagnetic resonance (EPR) spectroscopy}

All experiments were recorded using a home-built Q-band spectrometer capable of operating at frequencies between 35 and $36 \mathrm{GHz}$ with up to $150 \mathrm{~W}$ microwave power. The temperature was controlled by an Oxford Instruments (Abingdon, UK) cryostat by applying a constant flow of helium gas. Each sample was composed of around $10 \mathrm{mg}$ of the powder placed inside a standard $3 \mathrm{~mm}$ EPR tube. No degassing was performed. The $g$-tensors were calculated with respect to the phosphorus defect resonance at $10 \mathrm{~K}$. The $T_{1}$ relaxation time was measured using an inversion recovery sequence, with a 24 ns inversion $\pi$ pulse. The $T_{2}$ relaxation time was measured using a primary echo decay, with $\pi / 2-\tau-\pi$ pulse sequence, with pulses duration of $12 \mathrm{~ns}$ and $24 \mathrm{~ns}$, respectively. In each case, the minimum dead-time of the spectrometer was 688 ns. Both measurements were done at the field corresponding to the centre of the echo-detected EPR spectrum. Phenomenological models with two recovering exponents ( $T_{1}$ relaxation) or a stretch exponent ( $T_{2}$ relaxation) were used for data analysis. Such models were chosen based on their simplicity and the ability to reproduce the experimental data. For 
$T_{1}$ relaxation estimation, the two time constants extracted from a biexponential fit to an inversion recovery data are given as $\tau_{\text {fast }}$ and $\tau_{\text {slow }}$ while $A_{\text {fast }}, A_{\text {slow }}$ are the corresponding contributions of each of the time constants. For interpretation of transverse relaxation data, a stretch-exponent model was fitted with a time constant $t_{\mathrm{m}}$ and stretching factor $b$.

\section{Magic-angle spinning NMR}

Magic-angle spinning (MAS) NMR spectra were recorded using a Bruker $600 \mathrm{MHz}$ wide-bore magnet, operating at $119 \mathrm{MHz}\left({ }^{29} \mathrm{Si}\right.$ Larmor frequency). A $3.2 \mathrm{~mm}$ zirconia rotor with a Teflon cap was used. The sample was spun at $10 \mathrm{kHz}$ at room temperature. Each spectrum was acquired as the average of 256 scans with $700 \mathrm{~s}$ repetition time (total acquisition time: $2 \mathrm{~d}$ $1 \mathrm{~h})$. The nutation frequency of the excitation pulse $(2.5 \mu \mathrm{s})$ corresponded to $100 \mathrm{kHz}$ and 32 '768 complex points were acquired.

\section{Transmission electron microscopy (TEM)}

For the TEM investigation, the material was dispersed in ethanol and a few drops of the suspension were deposited onto a perforated carbon foil supported on a copper grid. After evaporation of the ethanol, the grid was mounted on the single tilt holder of the microscope. The imaging was performed using a Tecnai F30 (ThermoFisher) microscope, operated at an acceleration potential $\mathrm{U}_{\mathrm{ACC}}=300 \mathrm{kV}$ (field emission gun, $\mathrm{FEG}$ ). The particles' size distribution was analysed using ImageJ software $1.46 \mathrm{r}$ ( $\mathrm{NIH}$, Bethesda, Maryland USA).

\section{$X$-ray diffraction}

X-ray diffraction (XRD) spectra were acquired using a Bruker AXS D8 Advance X-ray powder diffractometer using $\mathrm{Cu} \mathrm{K} \alpha$ radiation. The $\mathrm{X}$-ray source was operated at $40 \mathrm{kV}$ voltage and $30 \mathrm{~mA}$ current. Each point in the spectrum was acquired for $1 \mathrm{~s}$. Crystal sizes were determined by fitting the main diffraction peak $\left(\sim 28^{\circ}\right)$ with the Topas 4 (Bruker) software and using the Scherrer equation. 


\section{SQUID magnetometry}

The magnetic susceptibility as a function of temperature $\chi(T)$ was measured in the $2.5-300 \mathrm{~K}$ range at a constant magnetic field $\left(B_{0}=1 \mathrm{~T}\right)$ by means of a commercial 7-T MPMS XL SQUID magnetometer (Quantum Design, San Diego, USA). In selected cases, also the field dependent magnetization in the 0-7 T range was measured at 2 and $5 \mathrm{~K}$. Between 20 and $60 \mathrm{mg}$ of sample were placed in a $1 \mathrm{~cm}$ long gelatine capsule and compressed with a cotton ball. The magnetization data were processed using an in-house developed MATLAB script. A spurious contribution from molecular oxygen was subtracted and the data below $40 \mathrm{~K}$ were inverted to obtain the linear slope for a magnetization calculation. Further details about magnetometry measurements are given in the supplementary information (see Fig. S1).

\section{Results}

The collective information about the physical properties of each silicon sample is shown in Table 1. The primary particle size distribution in each sample was confirmed by transmission electron microscopy (TEM) imaging as shown in Fig. 1. According to the information provided by the manufacturer, sample US 20-30 should have a primary particle size distribution between 20 and $30 \mathrm{~nm}$, however, in the particular batch that was received the size was found to be $55 \pm 12 \mathrm{~nm}$ (expressed as mean diameter \pm standard deviation). All samples exhibited a narrow size distribution except for sample AA 1-20, where the primary particle size varied between $100 \mathrm{~nm}$ and $25 \mu \mathrm{m}$.

The quantification of the mean crystallite size was obtained by XRD. An overview of the XRD spectra is shown in Fig. 2 and the zoomed-in spectra of each peak have been included in the supplementary information (Fig. S10). The crystallite sizes calculated from the patterns are summarized in Table 1. 
To obtain the microwave frequency corresponding to the maximum polarization transfer, the sweep spectra (also called DNP profiles, i.e., the dependence of the signal enhancement on the microwave frequency) were recorded. Prior to irradiation at each microwave frequency, the magnetization was saturated with a train of 32 radio-frequency pulses. Each point in the profile was recorded after $30 \mathrm{~min}$ of microwave irradiation. The polarization was measured with a set of four pulses with CYCLOPS phase cycling and a flip angle of about $3^{\circ}$. The DNP profiles for all samples are shown in Fig. 3. Each data set was normalized to the maximum absolute intensity and plotted over the same frequency range.

The DNP build-up time was recorded by continuous microwave irradiation ( $200 \mathrm{~mW}$ output power) for up to $24 \mathrm{~h}$ at a temperature of $T=3.5 \mathrm{~K}$. For samples AA 1-20, AA 50, US 20-30 and $\mathrm{SiP}$, the signal intensity over time was recorded by applying a small tip-angle excitation and collecting the free induction decay (FID) signal every $20 \mathrm{~min}$, overall resulting in more than 60 data points per build-up curve. Due to the low polarization enhancement, this was not possible for samples AA 1-5, AA 100 and US 1-3, for which each point in the build-up curve was measured with a hard $\pi / 2$ pulse and the sample was continuously repolarized (see Fig. 4). Consequently, the number of points in the build-up curve for these samples (AA 1-5, AA 100 and US 1-3) was restricted to less than ten. The characteristic time constants of the buildup were extracted with a mono- or biexponential fit to the experimental data (Table 1), depending on the number of points acquired.

The relaxation time has been assessed based on the magnetization decay after transfer of the silicon samples to the detection magnet (see Table 1). Because of the low enhancement, the room-temperature relaxation could not be recorded for samples AA 1-5, AA 100 and US 1-3. The electron relaxation was recorded at Q-band $(1.3 \mathrm{~T}, 35 \mathrm{GHz}$ microwave frequency, the corresponding echo-detected EPR spectra are given in the supplementary information, Fig. 
S2). The extracted relaxation-time constants are summarized in Table 2 . The full data sets including fits are provided in Fig. S4 and S5. The effect of temperature (10 K and $100 \mathrm{~K})$ on $T_{1}$ and of the excitation bandwidth (length of excitation pulse recorded at $\mathrm{T}=100 \mathrm{~K}$ ) on $T_{2}$ has been included in the supplementary information (Figures S6 and S7).

The absolute concentration of defects in the samples was determined using a SQUID magnetometer. Although the SQUID measurements cannot differentiate between different paramagnetic species in the sample (e.g. surface defect and contamination from paramagnetic impurities like iron or copper), it provides a reliable estimate of the absolute concentration of paramagnetic defects. Such quantification is not easily achievable using EPR spectroscopy as surface $\mathrm{Pb}$ defects have an average $g$-tensor value $(g \approx 2.0059)$ very close to organic radicals ( $g \approx 2.0025-2.0097$ ), and hence doping the sample with a known amount of exogenous radicals for calibration purpose would result in overlapping resonances from the two paramagnetic species (i.e. Pb defect and exogenous probe). The results of the estimation of the concentration of paramagnetic defects are summarized in Table 3. Values are given with respect to sample size (defects/g), particle surface area (defects/cm2), number of defects per particle (defects/particle) and defects per surface silicon atom (defects/surface atom).

The presence of other paramagnetic impurities in the sample (besides surface defects) can be indirectly assessed through the measurement of nuclear relaxation, which is influenced by paramagnetic relaxation enhancement (PRE) through nearby paramagnetic centres. In particular, $T_{2} *$ relaxation is a sensitive measure of the presence of paramagnetic impurities. For this purpose, linewidths of the ${ }^{29} \mathrm{Si}$ NMR resonance at room temperature were compared. The measurements were made in the solid state with the sample rotating at the magic angle to remove contribution from the anisotropic interactions (chemical shift, magnetic 
susceptibility). The spectrum of each sample exhibited only a single peak corresponding to elemental crystalline silicon, except for sample AA 100, where also a resonance from an amorphous phase was observed. The spectra are plotted in Fig. 5. Further analysis of the NMR line shape for data recorded with a DNP polarizer $\left(B_{0}=3.4 \mathrm{~T}\right)$ has been included in the supplementary information (Fig. S9).

The time dependence of the NMR line during microwave irradiation was analyzed for samples AA 1-20 and US $20-30$ as they exhibited the largest ${ }^{29} \mathrm{Si}$ signal enhancement. The linewidth was determined by a fit with a single Lorentzian curve. An example of changes in the linewidth during microwave irradiation together with a plot of the line width as a function of the polarization build-up time are shown in Fig. 6 .

The effect of microwave frequency modulation on the maximum ${ }^{29} \mathrm{Si}$ signal enhancement was studied by progressively changing the frequency or bandwidth of the modulation function (chain-saw) using an AA 1-20 sample. The change in the DNP profile and in the build-up curves of the nuclear polarization are presented in Fig. 7. In addition, the dependence of the enhancement factor on the modulation frequency and the bandwidth of the modulation are characterized.

Moreover, size dependent spin dynamics was also studied in the AA 1-20 sample which was subject to particle size separation. The dependence of the polarization build-up, maximum enhancement and NMR linewidth on the particle size were evaluated and the results are shown in Fig. 8. 


\section{Discussion}

EPR spectra of all examined samples show a single resonance arising from the Pb defects near the surface corresponding to an averaged $g$-factor of $g \approx 2.0059$. No signs of other paramagnetic defects or contributions from metal ions (apart from SiP, where a resonance from phosphorus defects are expected) could be identified in the EPR spectra. The EPR resonance in all samples showed a weak asymmetry, which can be attributed to residual heterogeneity of the interaction tensors. However, the EPR measurement was performed at a relatively low frequency $(35 \mathrm{GHz})$ which imposes a limitation on the detection of several paramagnetic spin species. The paramagnetic defects in silicon are characterized by similar $g$ tensor values ${ }^{30,36}$ and hence are difficult to disentangle at Q-band frequencies. Possible enhancement in a spectral resolution could be obtained by taking the measurement at higher field/frequency. Comparison between the EPR spectra recorded at $10 \mathrm{~K}$ and $100 \mathrm{~K}$ did not show any significant difference (see Fig S2, supplementary information), implying that the linewidths of the spectra are determined by the dispersion of $g$ - and hyperfine tensors rather than the transverse relaxation. The latter would be an indicator of the presence of paramagnetic forms of metal ions, characterized by much broader EPR lines. Moreover, the Raman spectra (supplementary information, Fig. S11) also did not show signs of contributions from other forms of siliconized material (e.g. silicon carbide) overall suggesting the high purity of the silicon particles. Some pronounced asymmetry in the EPR resonance in AA 100 samples has been observed, which can be attributed to the presence of the amorphous phase ${ }^{46}$ as seen in XRD and MAS NMR data (Figs. 2 and 5). This asymmetry in the EPR line translates into an asymmetry in the DNP profile (Fig. 3), which for sample AA 100, leads to significantly larger absolute enhancement recorded at the negative DNP peak. However, due to the overall low polarization enhancement of this sample, it is difficult to pinpoint the exact nature of this 
observation. Further work is needed to elucidate the exact DNP mechanisms (solid effect, cross effect, thermal mixture) leading to the observed polarization enhancement. Currently, an analysis of the sweep spectrum shape at different temperatures is used to distinguish between solid effect/cross effect DNP contributions. However, considering the length of the observed build-up times, such an experiment would be difficult to foreseen using current instrumentation due to limited time $(\approx 24 \mathrm{~h})$ of which the polarizing system can be run at low temperature.

The efficiency of polarization enhancement is given by the interplay of coherent (i.e. microwave irradiation) and incoherent (relaxation) spin dynamics, which in turn are affected by the properties of the examined material. In particular, the relaxation properties (both electronic and nuclear) of the silicon material seem to be the most significant factors affecting the polarizability of the particles. Out of the seven examined samples, only four showed significant polarization enhancement (samples AA 1-20, AA 50, US 20-30 and SiP). These samples were characterized by longer electronic $T_{1}$ and $T_{2}$ relaxation time than samples that did not show significant DNP enhancement (samples AA 1-5, AA 100, US 1-3). Importantly, these differences in relaxation time are more pronounced at low temperature $(10 \mathrm{~K}$ compared to $100 \mathrm{~K}$ - supplementary information, Fig. S6) indicating that the relaxation analysis should be done at a temperature close to the DNP polarization temperature. Unfortunately, performing relaxation measurements at the exact polarization temperature was not possible due to technical limitations of the EPR instruments used. Moreover, influence of spectral and spin diffusion on the recorded relaxation rates could be observed by varying excitation bandwidth (by changing the duration of the microwave pulses used) and thus covering different 'portions' of the EPR spectrum, (supplementary information, Fig. S7). In particular, samples AA 1-20 and US 20-30 (both characterized with large maximum signal 
enhancement) showed a large difference in the echo-decay time constants under excitation with a small and large frequency bandwidth, indicating the potentially important role of spectral spin diffusion on the electron spin bath dynamics. Recent theoretical and experimental results ${ }^{47,48}$ pointed out that the electron spin bath depolarization during microwave irradiation might be an important parameter affecting spin dynamics during DNP. However, due to hardware limitations, such a detailed analysis was not possible for the investigated samples.

The relaxation rate of a paramagnetic centre is closely linked to its structural environment. In a diluted lattice of spin-0 nuclei (both ${ }^{28} \mathrm{Si}$ and ${ }^{30} \mathrm{Si}$ are spin- 0 while ${ }^{29} \mathrm{Si}$ contributes to $4.7 \%$ of the isotopic abundance), the average hyperfine interaction with the neighboring nuclear spins is likely to be of a similar magnitude to the electron-electron dipolar coupling between two adjacent surface defects ${ }^{49}$. As such, the geometry of the surface defects is an important factor determining their relaxation properties. However, the recorded EPR spectra could not provide the necessary information as the samples have been measured as powders, hence the recorded spectra reflect an average over all the possible defect orientations. Consequently, the measured EPR spectra are relatively featureless and could not be used for fitting to obtain the distribution of hyperfine/g-tensors.

In order to gain more insight into the potential distribution of paramagnetic centres within the particles, the number of defects obtained from SQUID magnetometry was used to compute the ratio of the number of defects to total number of silicon atoms on the surface. Assuming an idealistic case of defects being only distributed on the surface of a particle, the ratio of defects per silicon atom at the surface should be less than 1 . As this ratio is greater than 1 (Table 3), it becomes clear that the surface defects cannot be assumed to create a monolayer on the particle surface but are rather dispersed in a multilayer geometry. This 
effect might be further exaggerated if there is some degree of surface porosity that enhances the effective surface area. Furthermore, for more in-depth analysis, the results of the ratio of defects/surface atom should be combined with the results of electronic $T_{2}$ relaxation. The dephasing of transverse relaxation (given by $T_{2}$ relaxation time) is enhanced by strong dipolar coupling with neighboring electron spins. As such, if the defects are located only at the surface, it is likely that transverse relaxation is faster, resulting in a shorter $T_{2}$ relaxation time constant. Correspondingly, if the defects are dispersed up to some depth below the $\mathrm{Si} / \mathrm{SiO}_{2}$ interface, the $T_{2}$ relaxation is not enhanced despite the large ratio of defects/surface. Bearing this in mind, samples AA 1-5 and US 1-3, which showed weak ${ }^{29}$ Si signal enhancement, are characterized with a large ratio of defects per surface atom (3.2 and 1.95, respectively) and showed the fastest electronic $T_{2}$ relaxations. At the same time, sample AA 1-20, which also has a comparably large ratio of defects/surface atom (2.8) showed a large signal enhancement and long electronic $T_{2}$ relaxation. This might indicate that in the case of the AA 1-20 sample, the defects are potentially 'dispersed' throughout some depth below the surface of the particles, while for samples AA 1-5 and US 1-3 the defects are only spread close to the surface. Foremost, this analysis shows the importance of defect distribution and geometry and that the absolute defect density is not a representative measure of silicon particle polarizability. Interestingly, a large polarization enhancement was observed for samples covering a broad range of sizes, from $50 \mathrm{~nm}$ (AA 50, US 20-30) to $10 \mu \mathrm{m}$ (AA 1-20). In particular, sample US 20-30 presented almost the same polarization value and roomtemperature nuclear $T_{1}$ relaxation despite the difference in radius by a factor of $\approx 20$. These results can also be explained by analyzing the defects-to-surface atom ratio. While for large, micrometer-size particles, the ratio can be large without compromising nuclear relaxation, it should be minimized for small, nanometre-size particles to prevent leakage of polarization 
due to rapid ${ }^{29} \mathrm{Si}$ nuclear relaxation by coupling to nearby surface defects. Samples AA 50 and US 20-30 (both $50 \mathrm{~nm}$ diameter) have the defects-to-surface atom ratio of 0.027 and 0.013 and both samples showed significant DNP enhancement. At the same time, sample AA 100 (100 $\mathrm{nm}$ diameter) was characterized by a defects-to-surface atom ratio of 0.14 , a factor of $\approx$ 10 larger than other nanometre-size particles and showed almost no ${ }^{29} \mathrm{Si}$ signal enhancement. Similar observations have been reported for hyperpolarization of micro- and nanodiamonds, which exhibit a similar morphology of surface defects ${ }^{50-53}$. The results of an analysis of the effect of defects concentration on the overall polarization dynamics are in line with results obtained with samples of organic solvent doped with the exogenous radicals ${ }^{54-57}$. Similarly to those reports, the results presented here showed a non-linear dependence of maximum achievable ${ }^{29} \mathrm{Si}$ nuclear polarization on paramagnetic centres' concentration. In particular, the optimal conditions for maximum signal enhancement are given by the superposition of radical concentration, isotopic ratio of the nuclear spin bath and the external magnetic field strength. The majority of these parameters are not easily adjustable in case of crystalline silicon particles as they are largly affected by the manufacturing approach.

The mean crystallite size did not show a clear correlation with the maximum achievable polarization nor with nuclear $T_{1}$ relaxation. The value of the mean crystallite size reported here for the AA 1-20 sample is an order of magnitude smaller than in previous reports for the same sample $\mathrm{e}^{12}$. Most likely this is to be attributed to the production differences.

The time dependence of the NMR linewidth during the microwave irradiation is indicative of polarization transfer from the surface into the core of the particle. As the nuclei located closest to the surface defects are polarized first, they also are characterized by the largest coupling to the paramagnetic defects. This leads to large $T_{2}{ }^{*}$ relaxation enhancement, which results in broadening of the NMR line. As the nuclear polarization is transferred into the core 
of a particle (through ${ }^{29} \mathrm{Si}$ nuclear spin diffusion), the bulk nuclei that are further away from the surface and hence do not experience such strong coupling to the surface defects are contributing to the narrow NMR line (Fig. 6). Examples of NMR lines for different samples and irradiation times, including the corresponding fits are given in Fig. S13, supplementary information. The most pronounced change in the NMR linewidth was observed for sample AA 1-20, which also had the largest number of defects per particle of all examined samples. The change in the NMR linewidth is almost not present for sample US 20-30, which has significantly fewer surface defects compared to sample AA 1-20. This makes it likely that the line broadening arises from the coupling to the surface defects. Interestingly, the linebroadening effect is less pronounced for sample AA 1-20, which has been size-separated and limited to average particle size $(A P S) \approx 400 \mathrm{~nm}$. In this case, the NMR line shape is still relatively broad at the end of the irradiation period $(\approx 2.5 \mathrm{kHz}$ versus $\approx 1 \mathrm{kHz}$ for $A A 1-20$ with unrestricted APS), which may be attributed to the large mechanical stress and resulting poor crystallinity during the milling process. This statement is further supported by the MAS NMR data recorded for the size-separated AA 1-20 sample (supplementary information, Fig. S12), where progressive line broadening is observed with decreasing APS.

The modulation of the microwave field has been shown to enhance significantly the maximum achievable polarization ${ }^{58}$. If the modulation is performed with a frequency higher than the electronic spin-lattice relaxation rate $R_{1}=1 / T_{1}$, the excitation bandwidth of the microwave field can be efficiently enlarged. Overall, a maximum polarization gain of about two was achieved compared with irradiation at a fixed frequency (Fig. 7). No difference in the buildup time was found between the two irradiation schemes, probably due to the dominating effect of the nuclear spin diffusion in the transfer of the polarization from the surface to the 
core of the particle. These results are in line with the previously published data ${ }^{12}$ recorded at $B_{0}=2.9 \mathrm{~T}$ and $T=3.5 \mathrm{~K}$.

In order to obtain more insight into size-dependent spin dynamics in silicon particles, the sample AA 1-20 was size separated into six batches, with APS ranging from $10 \mu \mathrm{m}$ to $400 \mathrm{~nm}$ (see Fig. 8). As the particles have been prepared identically (ball milling of a silicon ingot), this approach facilitates reliable comparison of the effect of particles' size on the observed ${ }^{29} \mathrm{Si}$ signal enhancement. Prior to recording the data for particles with different APS, a DNP profile (sweep spectrum) was recorded for a batch with APS $=0.5 \mu \mathrm{m}$ (see Fig S8, supplementary information). No change in the microwave frequency corresponding to the maximum polarization transfer was observed for particles with APS $=0.5 \mu \mathrm{m}$ as compared to AA 1-20 sample. The recorded build-up curves showed a distinctive change in the polarization buildup dynamics for particles with APS below $1.5 \mu \mathrm{m}$ with significantly reduced build-up time. The analysis with a biexponential fit to the build-up curves showed a change in the contributions of the two time constants (referred to as "fast" and "slow" time constant, respectively). For particles with APS $=1.1 \mu \mathrm{m}$ and larger, the build-up dynamic is dominated by a slow time constant which represents the transfer of the ${ }^{29} \mathrm{Si}$ polarization due to nuclear spin diffusion, while for particles with APS $=0.7 \mu \mathrm{m}$ and smaller, the polarization build-up is given by a fast time constant. This indicates that the small particles are polarized mostly by the direct coupling with the surface defects. In line with the data of the build-up dynamics for particles with a decreasing size, a similar observation was made for the maximum achievable polarization enhancement. Overall, a decrease in the ${ }^{29} \mathrm{Si}$ signal enhancement by a factor of three was observed when reducing the APS from $10 \mu \mathrm{m}$ to $400 \mathrm{~nm}$. Moreover, a pronounced difference in ${ }^{29} \mathrm{Si} \mathrm{NMR} \mathrm{line} \mathrm{shape} \mathrm{was} \mathrm{observed,} \mathrm{with} \mathrm{increased} \mathrm{linewidth} \mathrm{for} \mathrm{decreasing} \mathrm{APS.}$ Such a behaviour can be attributed to the increasing concentration of surface defects in 
respect to the particles' size resulting in enhanced $T^{*}{ }_{2}$ relaxation. This observation was further confirmed with MAS NMR ${ }^{29} \mathrm{Si}$ spectra recorded for a batch with APS $=0.4 \mu \mathrm{m}$, which also showed a marked increase in the linewidth compared to spectrum recorded for particles with APS $=1.1 \mu \mathrm{m}$ (see Fig. S12, supplementary information). The size-dependent analysis of the DNP efficiency showed, in line with the data presented previously for particles obtained from other sources, that the concentration of surface defects has to be tuned with respect to the particles' size. The large concentration of defects measured in sample AA 1-20 (see Table 3) was compensated by its large APS. However, as the APS was reduced, the efficiency of the polarization transfer from the surface defects to the ${ }^{29} \mathrm{Si}$ nuclei was diminished due to the increased nuclear $T_{1}$ relaxation.

\section{Conclusion}

We have demonstrated that endogenous surface defects in commercially available silicon micro- and nanoparticles can be used for ${ }^{29} \mathrm{Si}$ nuclear polarization enhancement by using direct ${ }^{29} \mathrm{Si}$ DNP. The electronic and nuclear relaxation properties and the surface-defect density correlates positively with the maximum achievable ${ }^{29} \mathrm{Si}$ nuclear polarization. Foremost, optimal physical and chemical conditions, including surface-defect concentration adjusted to the particle size, are necessary to achieve a high level of polarization enhancement. Among the examined samples, simple mechanical milling of the samples was sufficient to provide large, micrometre-size particles with the desired properties, while the application of the same method to produce small nanometre-size particles resulted in increased defect concentration and hence low polarization enhancement. Laser-assisted etching was found to be the most suitable production method to obtain nanometre-size silicon particles, which exhibit large maximum achievable ${ }^{29} \mathrm{Si}$ nuclear polarization and long 
room-temperature nuclear relaxation $T_{1}$ time. Further studies should focus on incorporating these finding into the production process to obtain particles with enhanced DNP properties. 


\section{Acknowledgements}

Financial support by the Swiss National Science Foundation (SNF grants 205320_16324, 206021_170729, 320030_153014, 200021_149707 and 200020_169879) is gratefully acknowledged. The authors are thankful to ScopeM, ETH Zurich, for access to the imaging facilities and Frank Krumeich for collecting TEM images. Christoph Guntlin's effort is appreciated for ball milling of the SiP silicon sample. Thomas Bauer is thanked for setting up the MAS NMR experiment. Reinhard Kissner is acknowledged for recording the Raman spectra. 


\section{References}

1. Ashbrook, S. E. \& Dawson, D. M. NMR spectroscopy of minerals and allied materials. Nuclear Magnetic Resonance 45, (2016).

2. Ivády, V. et al. Theoretical model of dynamic spin polarization of nuclei coupled to paramagnetic point defects in diamond and silicon carbide. Phys. Rev. B - Condens. Matter Mater. Phys. 92, 1-18 (2015).

3. Morton, J. J. L. et al. Solid-state quantum memory using the $31 \mathrm{P}$ nuclear spin. Nature 455, 1085-1088 (2008).

4. Abe, E. et al. Electron spin coherence of phosphorus donors in silicon: Effect of environmental nuclei. Phys. Rev. B - Condens. Matter Mater. Phys. 82, 9-12 (2010).

5. Hoehne, F. et al. Submillisecond hyperpolarization of nuclear spins in silicon. Phys. Rev. Lett. 114, 1-6 (2015).

6. Atkins, T. M. et al. Synthesis of Long T 1 Silicon Nanoparticles for Hyperpolarized 29 Si Magnetic Resonance Imaging. ACS Nano 7, 1609-1617 (2013).

7. Lee, M., Cassidy, M. C., Ramanathan, C. \& Marcus, C. M. Decay of nuclear hyperpolarization in silicon microparticles. Phys. Rev. B - Condens. Matter Mater. Phys. 84, 33-35 (2011).

8. Abe, E. et al. Line Broadening and Decoherence of Electron Spins in PhosphorusDoped Silicon Due to Environmental 29 Si Nuclear Spins. Phys. Rev. B 82, 12120 (2010).

9. Witzel, W. M., Carroll, M. S., Morello, A., Cywinski, Lukasz \& Das Sarma, S. Electron spin decoherence in isotope-enriched silicon. Phys. Rev. Lett. 105, (2010).

10. Dementyev, A. E., Cory, D. G. \& Ramanathan, C. Dynamic Nuclear Polarization in Silicon Microparticles. Phys. Rev. Lett. 100, 127601 (2007).

11. Aptekar, J. W. et al. Hyperpolarized Long-T1 Silicon Nanoparticles for Magnetic Resonance Imaging. 1-5 (2009). doi:10.1021/nn900996p

12. Cassidy, M. C., Chan, H. R., Ross, B. D., Bhattacharya, P. K. \& Marcus, C. M. In vivo magnetic resonance imaging of hyperpolarized silicon particles. Nat. Nanotechnol. 8, 363-368 (2013).

13. Whiting, N. et al. Developing hyperpolarized silicon particles for in vivo MRI targeting of ovarian cancer. J. Med. Imag. 3, 036001 (2016).

14. Whiting, N. et al. Real-Time MRI-Guided Catheter Tracking Using Hyperpolarized Silicon Particles. Sci. Rep. 5, 12842 (2015).

15. Kwiatkowski, G. et al. Nanometer size silicon particles for hyperpolarized MRI. Sci. Rep. 7, (2017). 
16. Keshari, K. R. \& Wilson, D. M. Chemistry and biochemistry of $13 \mathrm{C}$ hyperpolarized magnetic resonance using dynamic nuclear polarization. Chem. Soc. Rev. 43, (2014).

17. Zou, J., Baldwin, R. K., Pettigrew, K. A. \& Kauzlarich, S. M. Solution synthesis of ultrastable luminescent siloxane-coated silicon nanoparticles. Nano Lett. 4, 11811186 (2004).

18. Vons, V. A. et al. Silicon nanoparticles produced by spark discharge. J. Nanoparticle Res. 13, 4867-4879 (2011).

19. Houle, F. A. Photostimulated desorption in laser-assisted etching of silicon. Phys. Rev. Lett. 61, 1871-1874 (1988).

20. Kuzmin, P. G. et al. Silicon Nanoparticles Produced by Femtosecond Laser Ablation in Ethanol : Size Control , Structural Characterization , and Optical Properties. 1526615273 (2010).

21. Mangolini, L., Thimsen, E. \& Kortshagen, U. High-Yield Plasma Synthesis of Luminescent Silicon Nanocrystals. Nano Le 5, 655-659 (2005).

22. Mangolini, L. \& Kortshagen, U. Plasma-assisted synthesis of silicon nanocrystal inks. Adv. Mater. 19, 2513-2519 (2007).

23. Kim, B., Hiroyuki, U., Tooru, F., Tadashi, I. \& Mamoru, S. Fabrication of nano-sized Si powders with a narrow size distribution by two-step milling. J. Mater. Res. 18, 13681373 (2003).

24. Dasog, M., Kehrle, J., Rieger, B. \& Veinot, J. G. C. Silicon nanocrystals and siliconpolymer hybrids: Synthesis, surface engineering, and applications. Angew. Chemie Int. Ed. 55, 2322-2339 (2016).

25. Nikolaou, P., Goodson, B. M. \& Chekmenev, E. Y. NMR hyperpolarization techniques for biomedicine. Chem. - A Eur. J. 21, 3156-3166 (2015).

26. Abragam, A. \& Goldman, M. Principles of dynamic nuclear polarisation. Rep. Prog. Phys. 41, 396-467 (1978).

27. Hovav, Y., Feintuch, A. \& Vega, S. Theoretical aspects of dynamic nuclear polarization in the solid state - The solid effect. J. Magn. Reson. 207, 176-189 (2010).

28. Newman, R. C. Defects in silicon. Reports Prog. Phys. 45, 1163-1210 (2000).

29. Watkins, G. D. Intrinsic defects in silicon. Mater. Sci. Semicond. Process. 3, 227-235 (2000).

30. Caplan, P. J., Helbert, J. N., Wagner, B. E. \& Poindexter, E. H. Paramagnetic defects in silicon/silicon dioxide systems. Surf. Sci. 54, 33-42 (1976).

31. Khutsishvili, G. R. The Overhauser effect and related phenomena. Usp. Fiz. Nauk 71, 9-69 (1960).

32. Riikonen, J. et al. Endogenous Stable Radicals for Characterization of Thermally 
Carbonized Porous Silicon by Solid-State Dynamic Nuclear Polarization ${ }^{13} \mathrm{C}$ NMR. J. Phys. Chem. C 119, 19272-19278 (2015).

33. Guy, M. L., Van Schooten, K. J., Zhu, L. \& Ramanathan, C. Chemisorption of Water on the Surface of Silicon Microparticles Measured by Dynamic Nuclear Polarization Enhanced NMR. J. Phys. Chem. C 121, 2748-2754 (2017).

34. Cassidy, M. C., Ramanathan, C., Cory, D. G., Ager, J. W. \& Marcus, C. M. Radicalfree dynamic nuclear polarization using electronic defects in silicon. Phys. Rev. B 87, 161306 (2013).

35. Caplan, P. J., Poindexter, E. H., Deal, B. E. \& Razouk, R. R. ESR centers, interface states, and oxide fixed charge in thermally oxidized silicon wafers ESR centers, interface states, and oxide fixed charge in thermally oxidized silicon wafers. J. Appl. Phys. 50, 5847-5854 (1979).

36. Poindexter, E. H. \& Caplan, P. J. Characterization of Si/SiO2 Interface Defects by Electron Spin Resonance. Prog. Surf. Sci. 14, 201-294 (1983).

37. Dementyev, A. E., Cory, D. G. \& Ramanathan, C. High-field Overhauser dynamic nuclear polarization in silicon below the metal-insulator transition. J. Chem. Phys. 134, (2011).

38. Hayashi, H., Itahashi, T., Itoh, K., Vlasenko, L. \& Vlasenko, M. Dynamic nuclear polarization of S29i nuclei in isotopically controlled phosphorus doped silicon. Phys. Rev. B 80, 045201 (2009).

39. Itahashi, T., Hayashi, H., Rahman, M. R. \& Itoh, K. M. Optical and dynamic nuclear polarization of $29 \mathrm{Si}$ nuclei via photoexcited triplet states of oxygen-vacancy complexes in isotopically controlled silicon. 075201, 1-10 (2013).

40. Verhulst, A., Rau, I., Yamamoto, Y. \& Itoh, K. Optical pumping of Si29 nuclear spins in bulk silicon at high magnetic field and liquid helium temperature. Phys. Rev. B 71, 235206 (2005).

41. Lesage, A. et al. Surface enhanced NMR spectroscopy by dynamic nuclear polarization. J. Am. Chem. Soc. 132, 15459-15461 (2010).

42. Seo, H. et al. Hyperpolarized Porous Silicon Nanoparticles: Potential Theragnostic Material for 29Si Magnetic Resonance Imaging. ChemPhysChem 1-6 (2018). doi:10.1002/cphc.201800461

43. Hu, J., Whiting, N. \& Bhattacharya, P. Hyperpolarization of Silicon Nanoparticles with TEMPO Radicals. J. Phys. Chem. C 122, 10575-10581 (2018).

44. Brown, C. Particle-size distributions by centrifugal sedimentation. J. Phys. Chem. 48, 246-258 (1944).

45. Batel, M. et al. A multi-sample $94 \mathrm{GHz}$ dissolution dynamic-nuclear-polarization system. J. Magn. Reson. 214, 166-174 (2012). 
46. Weil, J. A. A review of the EPR spectroscopy of the point defects in a-quartz: The decade 1982-1992. The Physics and Chemistry of SiO2 and the Si-SiO2 Interface 2 (1992).

47. Hovav, Y. et al. The electron depolarization during dynamic nuclear polarization: Measurements and simulations. Phys. Chem. Chem. Phys. 17, 226-244 (2015).

48. Hovav, Y. et al. Effects of the electron polarization on uynamic Nuclear Polarization in Solids. Phys. Chem. Chem. Phys. 17, 6053-6065 (2015).

49. Browe, K. L. \& Headley, T. J. Dipolar interactions between dangling bonds at the (111)Si-SiO2 interface. Phys. Rev. B 34, 3610 (1986).

50. Casabianca, L. B., Shames, A. I., Panich, A. M., Shenderova, O. \& Frydman, L. Factors affecting DNP NMR in polycrystalline diamond samples. J. Phys. Chem. C 115, 19041-19048 (2011).

51. Bretschneider, C. O. et al. On The Potential of Dynamic Nuclear Polarization Enhanced Diamonds in Solid-State and Dissolution 13CNMR Spectroscopy. ChemPhysChem 17, 1-12 (2016).

52. Rej, E., Gaebel, T., Boele, T., Waddington, D. E. J. \& Reilly, D. J. Hyperpolarized nanodiamond with long spin-relaxation times. Nat. Commun. 6, 8459 (2015).

53. Kwiatkowski, G. et al. Direct hyperpolarization of micro- and nanodiamonds for bioimaging applications - Considerations on particle size, functionalization and polarization loss. J. Magn. Reson. 286, (2018).

54. Ardenkjaer-Larsen, J. H., Macholl, S. \& Jóhannesson, H. Dynamic nuclear polarization with trityls at $1.2 \mathrm{~K}$. Appl. Magn. Reson. 34, 509-522 (2008).

55. Jóhannesson, H., Macholl, S. \& Ardenkjaer-Larsen, J. H. Dynamic Nuclear Polarization of [1-13C]pyruvic acid at 4.6 tesla. J. Magn. Reson. 197, 167-175 (2009).

56. Hu, K. N., Bajaj, V. S., Rosay, M. \& Griffin, R. G. High-frequency dynamic nuclear polarization using mixtures of TEMPO and trityl radicals. J. Chem. Phys. 126, (2007).

57. Jahnig, F. et al. Dissolution DNP using trityl radicals at $7 \mathrm{~T}$ field. Phys. Chem. Chem. Phys. 19, 19196-19204 (2017).

58. Hovav, Y., Feintuch, A., Vega, S. \& Goldfarb, D. Dynamic nuclear polarization using frequency modulation at 3.34 T. J. Magn. Reson. 238, 94-105 (2014). 


\section{Figures}
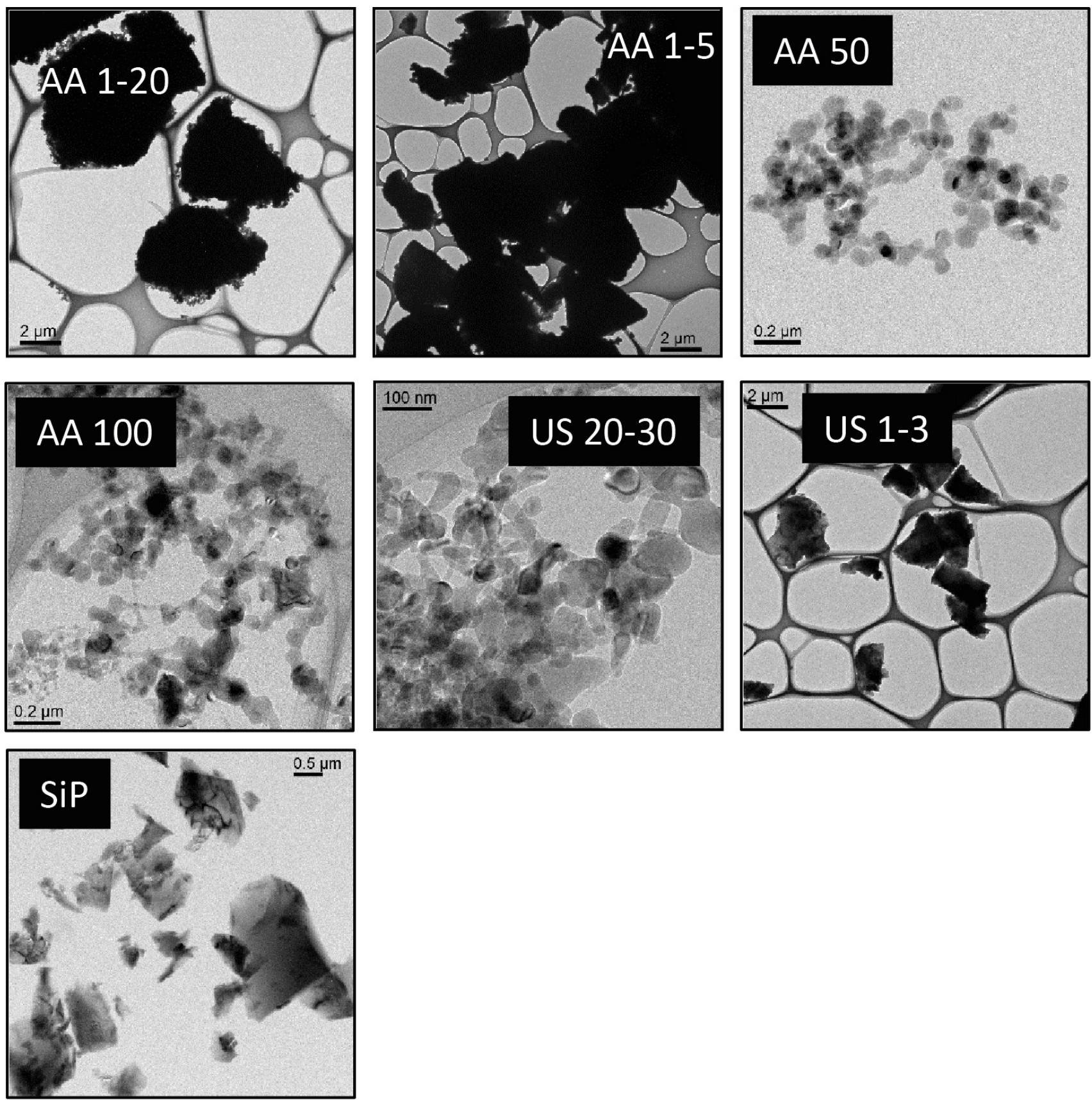

Figure 1. A series of representative TEM pictures illustrating the morphology of each of the examined samples (see Table 1). The scale has been adjusted according to the material size. 


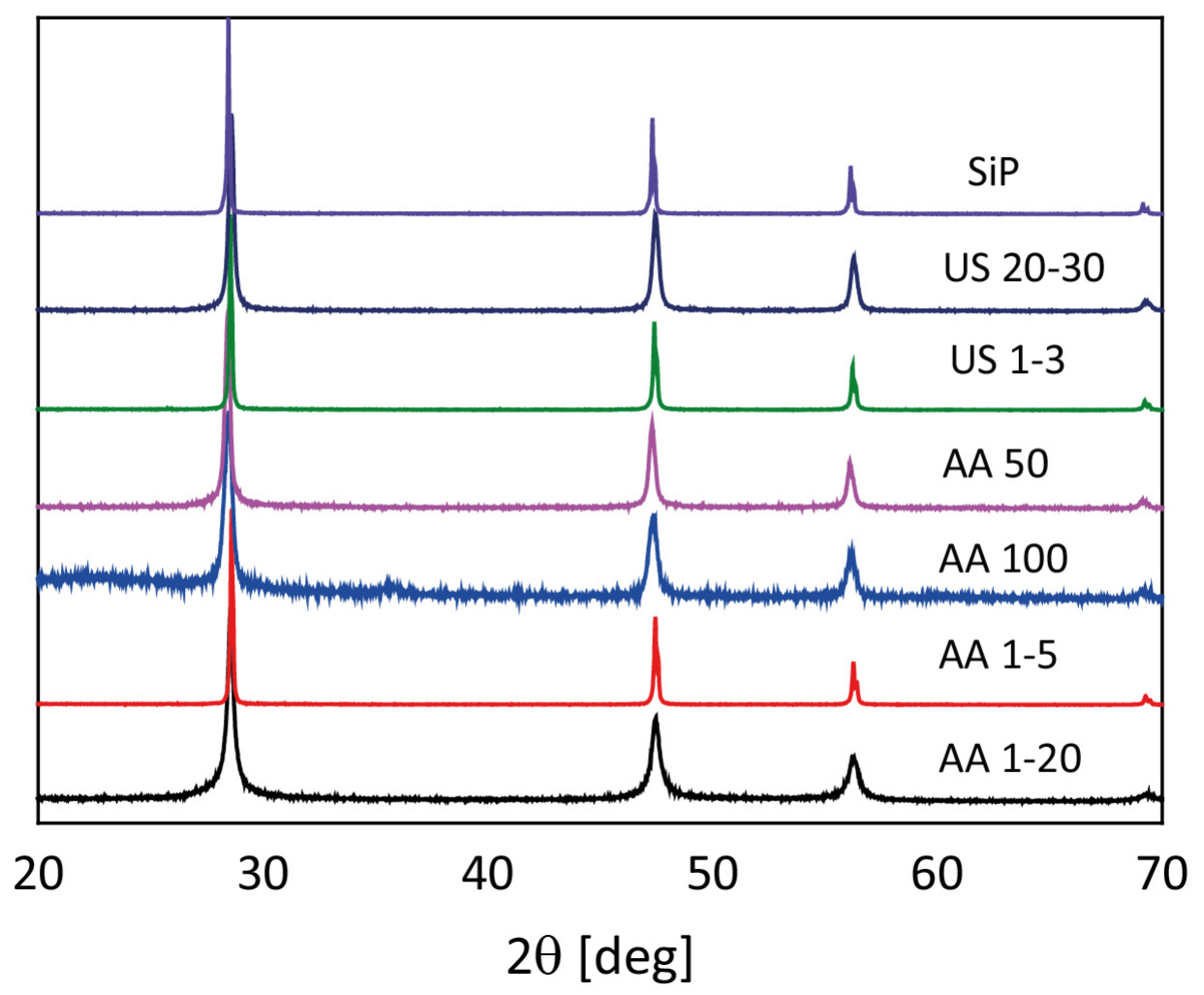

Figure 2. X-ray diffraction patterns of all examined samples. 

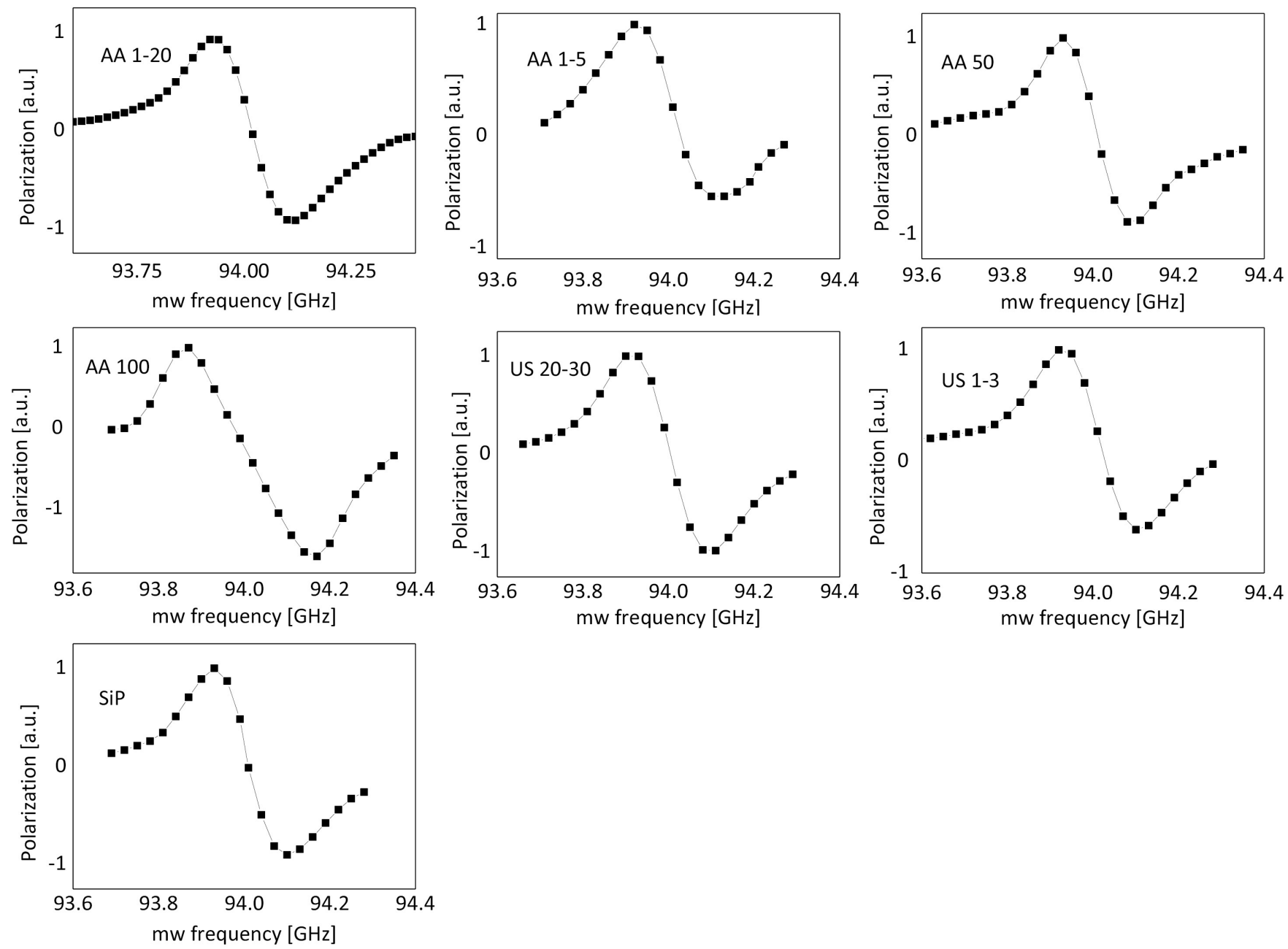

Figure 3. The sweep spectra (DNP profiles) of all examined samples, recorded at $T=3.5 \mathrm{~K}$. Each spectrum was normalized to its maximum signal intensity and plotted over the same range of microwave frequencies. 

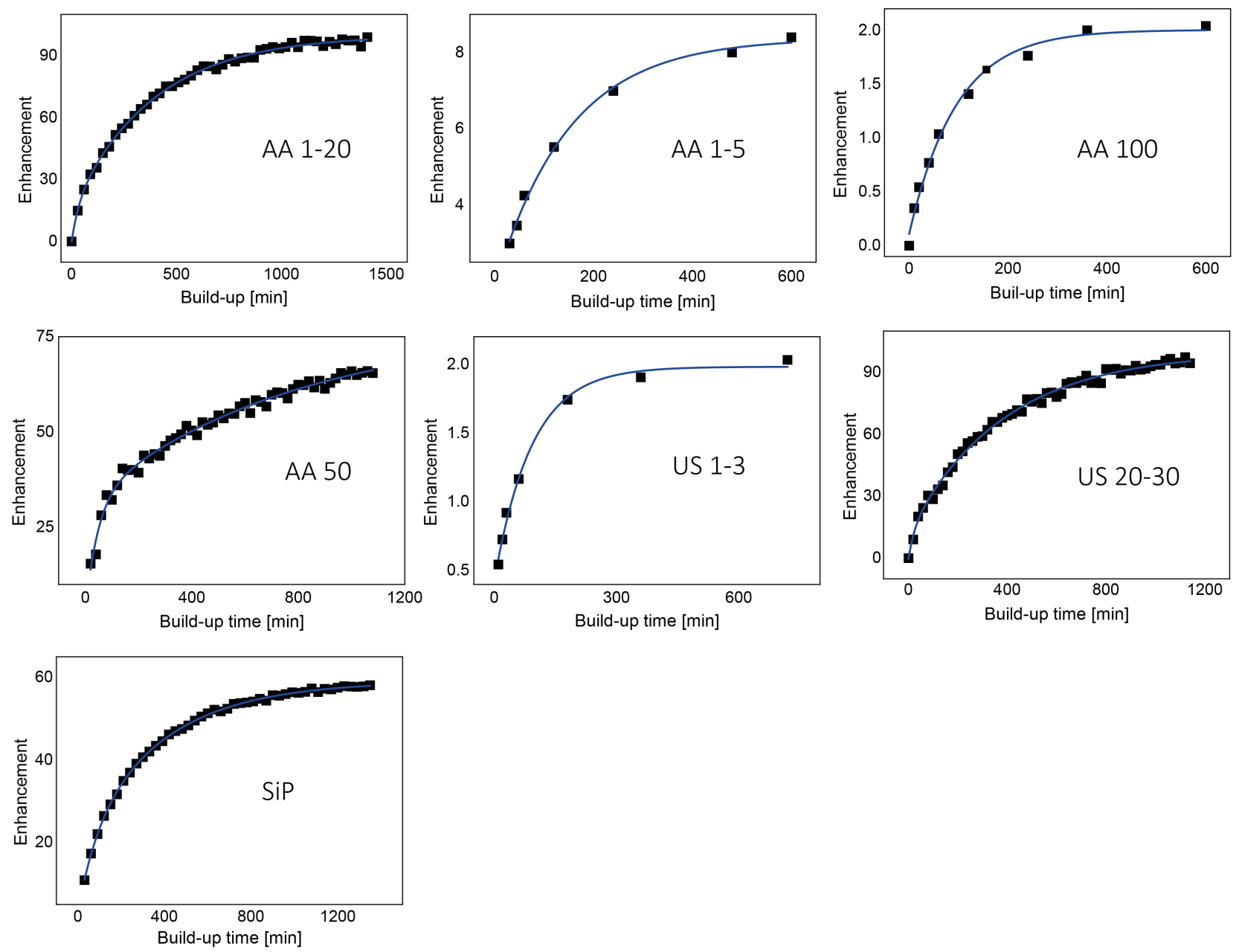

Figure 4. The build-up curves of ${ }^{29} \mathrm{Si}$ nuclear polarization build-up during continuous microwave irradiation recorded at $T=3.5 \mathrm{~K}$. Each sample was irradiated at the microwave frequency corresponding to the maximum positive ${ }^{29} \mathrm{Si}$ nuclear polarization enhancement, according to data presented in Fig. 3. The black squares represent the experimental data points while blue curves are the mono/biexponential fit to the data. 

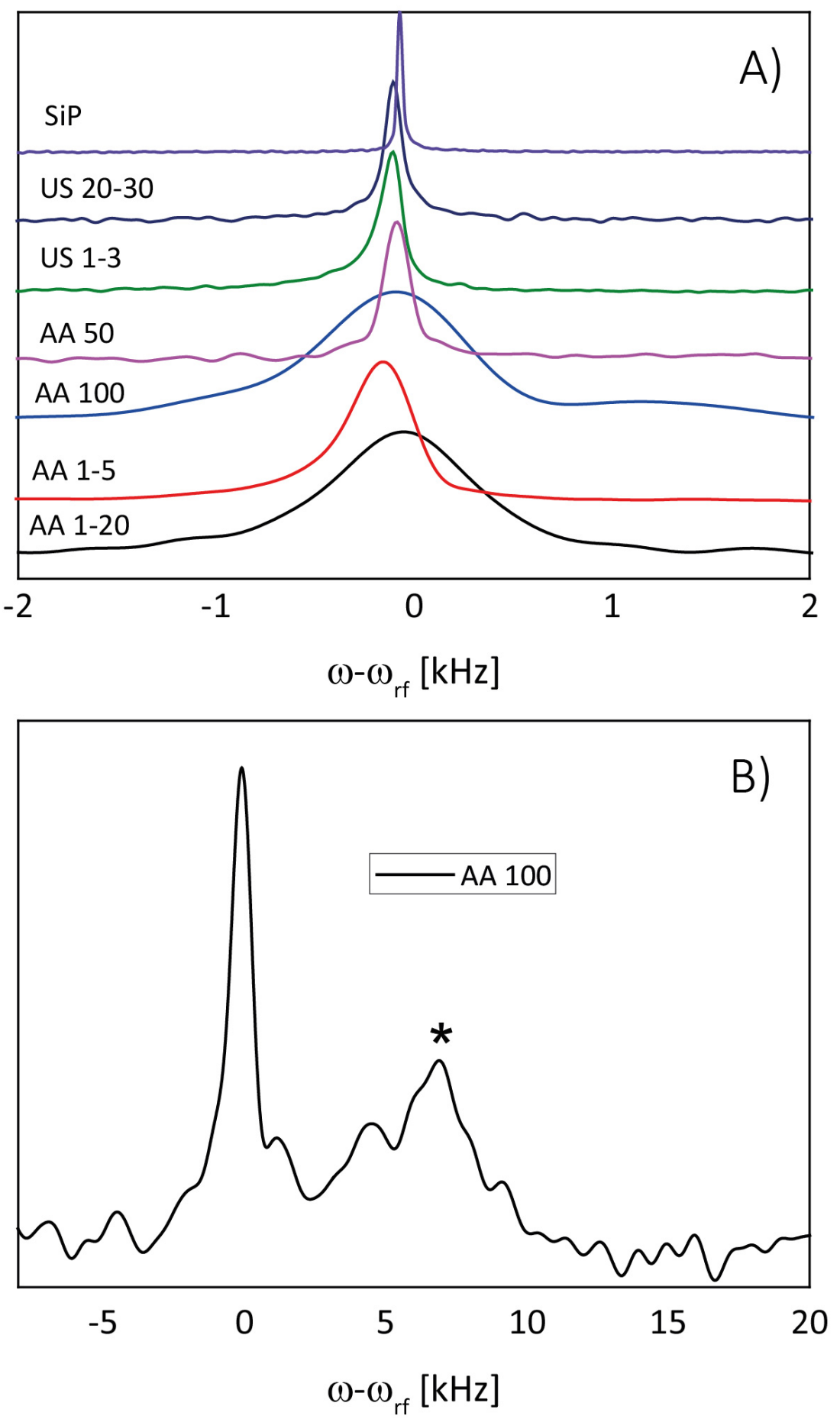

Figure 5. A) The MAS NMR spectra recorded at $B_{0}=14 \mathrm{~T}\left(\omega / 2 \pi\left({ }^{29} \mathrm{Si}\right)=119 \mathrm{MHz}\right)$ and room temperature. B). The zoom-in of the spectrum for sample AA 100 shows a resonance from an amorphous silicon phase (indicated by an asterisk). Each spectrum was normalized before plotting and shifted in vertical direction for enhanced visualization. 

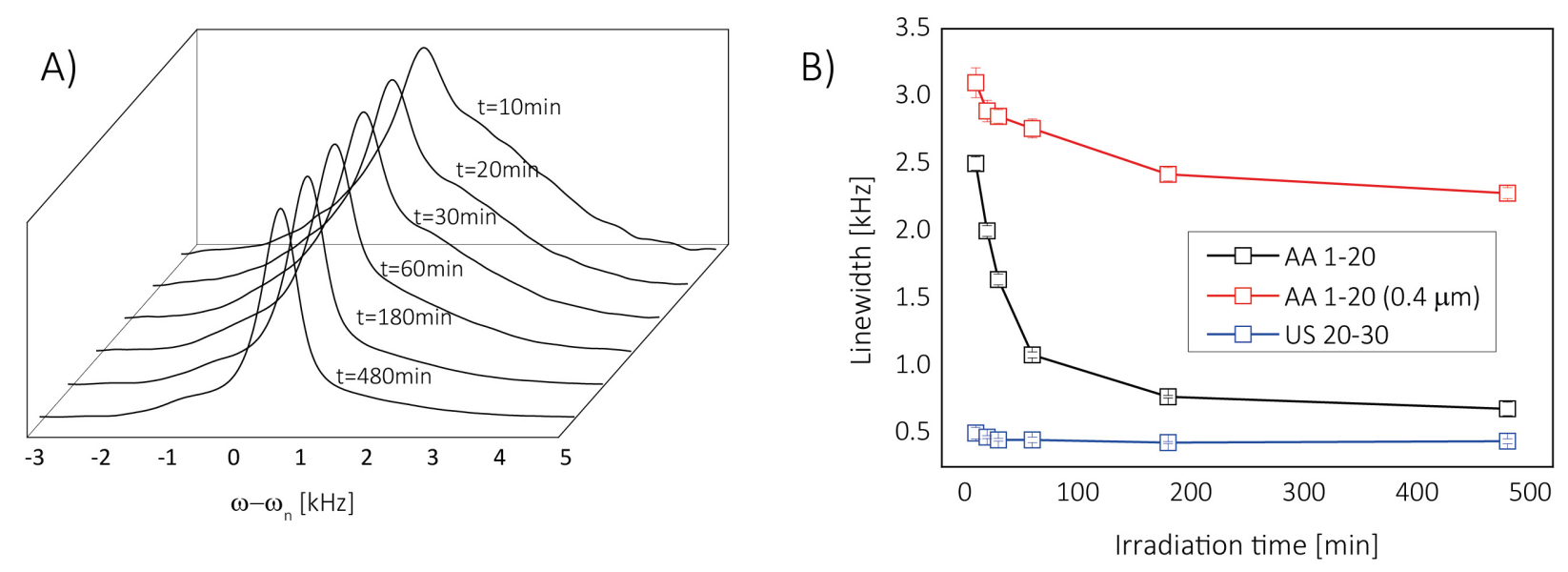

Figure 6. A) The NMR spectra of the AA 1-20 sample recorded dynamically during microwave irradiation at $T=3.5 \mathrm{~K}$. The spectra were collected with a hard radiofrequency pulse at the given time point. The sample was repolarized for each spectrum. Each spectrum was normalized to unity before plotting. B) The time dependence of the full width at half-height during microwave irradiation calculated for samples AA 1-20 (black), size-separated AA 1-20 with APS $\approx 0.4 \mu \mathrm{m}$ (red) and US 20-30 (blue). 

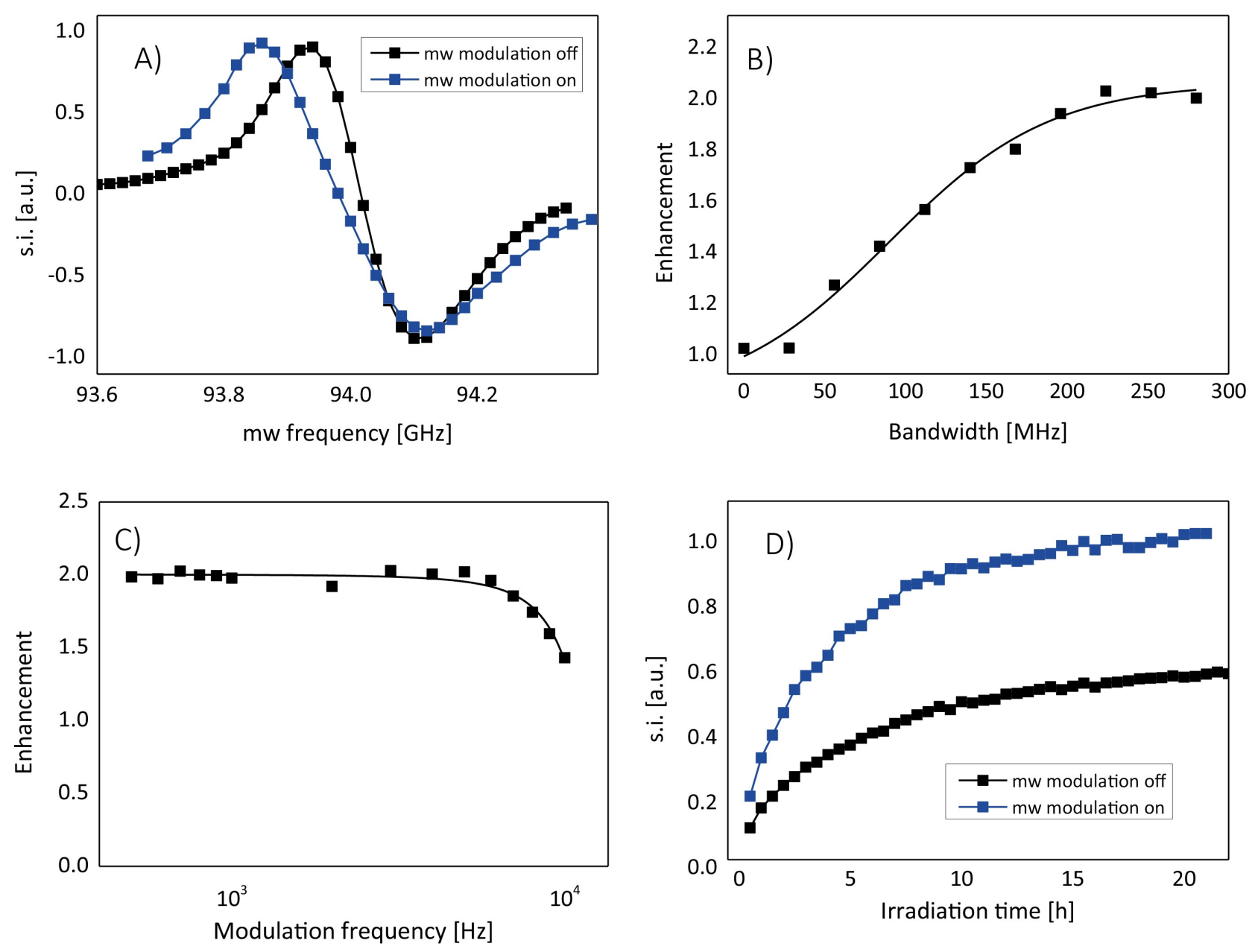

Figure 7. Effect of the microwave frequency modulation. A) Sweep spectra recorded without (black) microwave field modulation and with (blue) using $3 \mathrm{kHz}$ chain-saw modulation with $300 \mathrm{MHz}$ bandwidth. B) Signal enhancement as a function of modulation bandwidth and (C) modulation frequency. D) The build-up of ${ }^{29} \mathrm{Si}$ nuclear polarization with (blue) and without (black) microwave modulation. The data were recorded at $T=3.4 \mathrm{~K}$. 

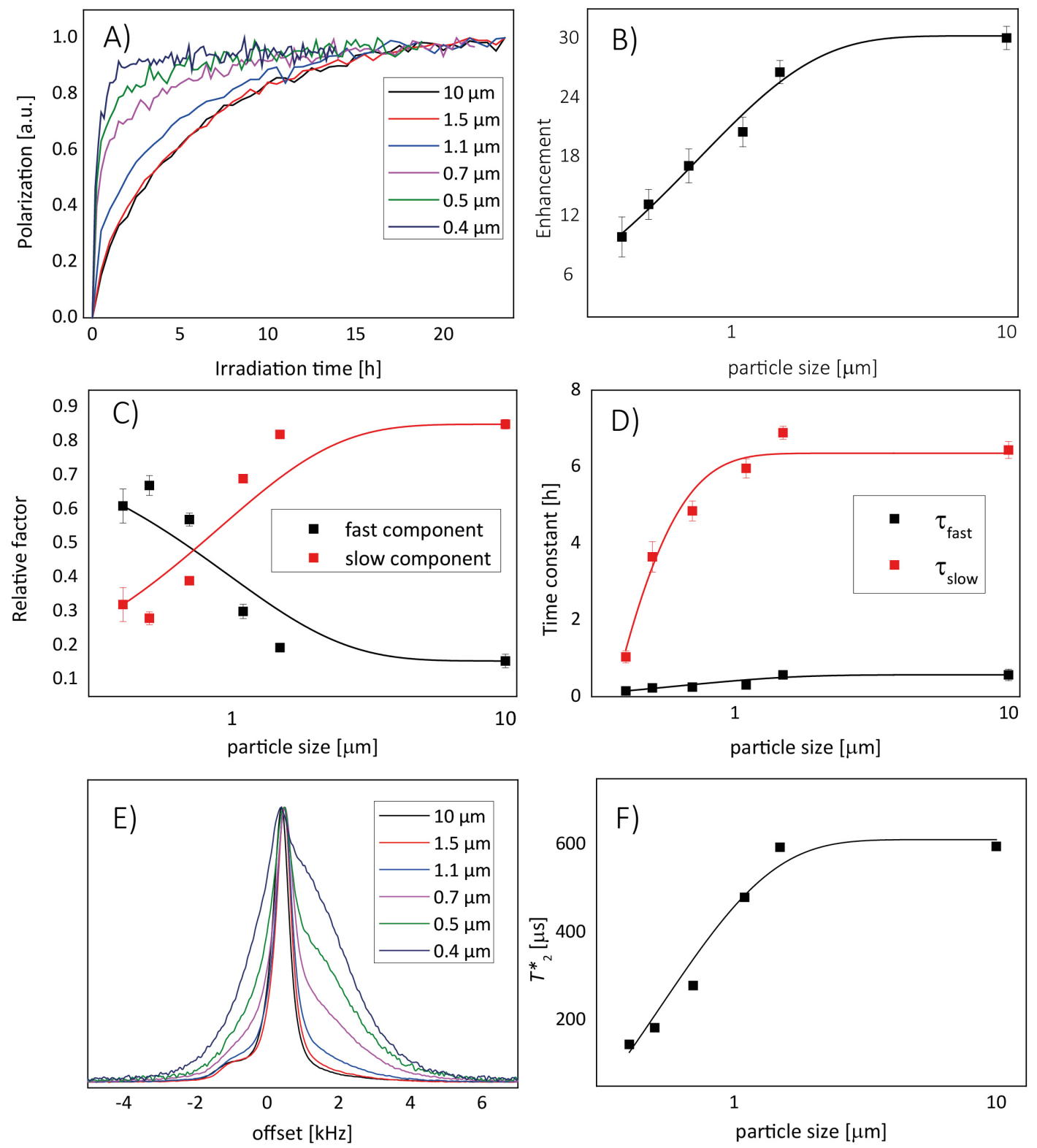

Figure 8. Size-dependent spin dynamics in the AA 1-20 sample. A) Build-up time curves recorded under continuous microwave irradiation. B) Corresponding enhancement at $3.5 \mathrm{~K}$. C) The relative contribution of each time constant presented and D) time constant extracted using a biexponential fit to polarization build-up data presented in (A). E) The NMR spectra recorded at $3.5 \mathrm{~K}$ and $\mathrm{F}$ ) the nuclear $T_{2}{ }^{*}$ time constant extracted from a monoexponential fit to the FID data. All data points are presented with errors when applicable. Solid lines in panels $\mathrm{B}, \mathrm{C}, \mathrm{D}, \mathrm{F}$ are for guidance only. The data were recorded at $T=3.4 \mathrm{~K}$. 
Tables

\begin{tabular}{|c|c|c|c|c|c|c|c|c|}
\hline Acronym & Method & APS & \multicolumn{2}{|c|}{$\begin{array}{l}\text { Build-up } \\
\text { time [min] }\end{array}$} & Enhancement & g value & $\begin{array}{c}T_{1} \text { at } R T \\
\text { [min] }\end{array}$ & $\begin{array}{c}\text { Mean } \\
\text { crystalline } \\
\text { size }[\mathrm{nm}]\end{array}$ \\
\hline AA 1-20 & $\begin{array}{c}\text { ball milled } \\
\text { crystalline ingot }\end{array}$ & $\begin{array}{c}1- \\
20 \mu \mathrm{m}\end{array}$ & $\begin{array}{r}24 \\
\pm 7\end{array}$ & $392 \pm 49$ & $29 \pm 8$ & $\begin{array}{l}2.0059 \pm \\
0.00007\end{array}$ & $45.5 \pm 3.2$ & 20.7 \\
\hline AA 1-5 & $\begin{array}{l}\text { metal basis, jet } \\
\text { milled }\end{array}$ & $1-5 \mu \mathrm{m}$ & \multicolumn{2}{|c|}{$133 \pm 14$} & $1.0 \pm 0.3$ & $\begin{array}{l}2.0059 \pm \\
0.00007\end{array}$ & / & 130 \\
\hline AA 100 & $\begin{array}{c}\text { plasma } \\
\text { synthesized }\end{array}$ & $100 \mathrm{~nm}$ & \multicolumn{2}{|c|}{$108 \pm 12$} & $1.0 \pm 0.3$ & $\begin{array}{l}2.0059 \pm \\
0.00007 \\
2.0022 \pm \\
0.00007\end{array}$ & / & 27.9 \\
\hline AA 50 & $\begin{array}{l}\text { laser synthesized } \\
\text { from vapor } \\
\text { phase }\end{array}$ & $50 \mathrm{~nm}$ & $\begin{array}{r}46 \\
\pm 9\end{array}$ & $772 \pm 150$ & $16.2 \pm 4.6$ & $\begin{array}{l}2.0059 \pm \\
0.00007\end{array}$ & $56.7 \pm 4.3$ & 41.9 \\
\hline US 1-3 & $\begin{array}{c}\text { plasma } \\
\text { enhanced } \\
\text { chemical vapor } \\
\text { depsition }\end{array}$ & $1-3 \mu \mathrm{m}$ & \multicolumn{2}{|c|}{$92 \pm 10$} & $1.0 \pm 0.3$ & $\begin{array}{l}2.0059 \pm \\
0.00007\end{array}$ & / & 115 \\
\hline US 20-30 & $\begin{array}{c}\text { laser gas phase } \\
\text { synthesis }\end{array}$ & $50 \mathrm{~nm}$ & \multicolumn{2}{|c|}{$229 \pm 5$} & $27.4 \pm 8.1$ & $\begin{array}{l}2.0059 \pm \\
0.00007\end{array}$ & $42.8 \pm 3.4$ & 42.7 \\
\hline SiP & $\begin{array}{c}\text { ball milled slicon } \\
\text { wafer }\end{array}$ & $2-6 \mu \mathrm{m}$ & $\begin{array}{c}85 \\
\pm \\
12\end{array}$ & $375 \pm 18$ & $8.1 \pm 2.4$ & $\begin{array}{c}2.0059 \pm \\
0.00007 \\
1.995 \pm 0.00007\end{array}$ & $130 \pm 11$ & 146.1 \\
\hline
\end{tabular}

Table 1. Collective data on physicochemical properties of the examined silicon powders 


\begin{tabular}{ccccccc}
\hline \multirow{2}{*}{ Material } & \multicolumn{2}{c}{$T_{1}$ relaxation } & \multicolumn{3}{c}{$T_{2}$ relaxation } \\
\cline { 2 - 7 } & $A_{\text {fast }}$ & $\tau_{\text {fast }}[\mathrm{ms}]$ & $A_{\text {slow }}$ & $\tau_{\text {slow }}[\mathrm{ms}]$ & $t_{\mathrm{m}}[\mu \mathrm{s}]$ & 6 \\
\hline \multirow{2}{*}{ AA 1-20 } & $0.5654 \pm$ & $1.411 \pm$ & $0.4346 \pm$ & $61.32 \pm$ & $1.631 \pm$ & $0.637 \pm 0.023$ \\
& 0.0027 & 0.041 & 0.0025 & 0.56 & 0.013 & \\
\hline \multirow{2}{*}{ AA 1-5 } & $0.7382 \pm$ & $0.193 \pm$ & $0.2618 \pm$ & $7.12 \pm$ & $0.187 \pm$ & $0.3762 \pm$ \\
& 0.0033 & 0.0046 & 0.0030 & 0.13 & 0.004 & 0.0023 \\
\hline \multirow{2}{*}{ AA-50 } & $0.7720 \pm$ & $1.233 \pm$ & $0.2280 \pm$ & $25.54 \pm$ & $1.63 \pm 0.01$ & $0.664 \pm 0.002$ \\
& 0.0033 & 0.023 & 0.0065 & 0.49 & & \\
\multirow{2}{*}{ AA-100 } & $0.7709 \pm$ & $1.37 \pm 0.03$ & $0.2291 \pm$ & $27.3 \pm 0.5$ & $1.133 \pm$ & $0.6532 \pm$ \\
& 0.0068 & 0.0065 & 0.029 & 0.0073 \\
\hline \multirow{2}{*}{ US 1-3 } & $0.7764 \pm$ & $0.631 \pm$ & $0.2236 \pm$ & $12.37 \pm$ & $0.275 \pm$ & $0.429 \pm 0.001$ \\
& 0.0038 & 0.012 & 0.0036 & 0.18 & 0.003 & $0.003 \pm$ \\
\multirow{2}{*}{ US 20-30 } & $0.5898 \pm$ & $3.382 \pm$ & $0.4107 \pm$ & $74.62 \pm$ & $2.962 \pm$ & $0.6269 \pm$ \\
& 0.0034 & 0.073 & 0.0030 & 0.85 & 0.023 & 0.0025 \\
\hline \multirow{2}{*}{ SiP } & $0.6565 \pm$ & $0.336 \pm$ & $0.3435 \pm$ & $18.4 \pm 0.8$ & $0.87 \pm 0.01$ & $0.5093 \pm$ \\
& 0.0040 & 0.023 & 0.0038 & 0.0018 \\
\hline
\end{tabular}

Table 2. EPR relaxation measurements of longitudinal $\left(T_{1}\right)$ and transverse $\left(T_{2}\right)$ relaxation. 


\begin{tabular}{ccccc}
\hline Material & [defects/g] & [defects/cm ${ }^{2}$ ] & [defects/particle] & [defects/surf. atom] \\
\hline AA 1-20 & $4.89 \cdot 10^{18}$ & $1.90 \cdot 10^{15}$ & $4.77 \cdot 10^{10}$ & 2.80 \\
\hline AA 1-5 & $1.12 \cdot 10^{19}$ & $1.09 \cdot 10^{15}$ & $1.36 \cdot 10^{10}$ & 3.20 \\
\hline AA 50 & $4.55 \cdot 10^{18}$ & $8.83 \cdot 10^{12}$ & $5.55 \cdot 10^{3}$ & 0.013 \\
\hline AA 100 & $2.45 \cdot 10^{19}$ & $9.51 \cdot 10^{13}$ & $2.39 \cdot 10^{5}$ & 0.14 \\
\hline US 20-30 & $9.60 \cdot 10^{18}$ & $1.86 \cdot 10^{13}$ & $1.17 \cdot 10^{4}$ & 0.027 \\
\hline US 1-3 & $1.14 \cdot 10^{19}$ & $6.64 \cdot 10^{14}$ & $3.00 \cdot 10^{9}$ & 1.957 \\
\hline SiP & $5.05 \cdot 10^{18}$ & $7.84 \cdot 10^{14}$ & $3.16 \cdot 10^{9}$ & 1.57 \\
\hline
\end{tabular}

Table 3. Defect concentrations determined with SQUID magnetometry. 